Pacific Journal of Mathematics

PSEUDO REGULAR ELEMENTS IN A NORMED RING 


\title{
PSEUDO REGULAR ELEMENTS IN A NORMED RING
}

\author{
RICHARD F. ARENS
}

Let $A$ be an algebra, and let $f$ be a linear mapping of $A$ into some normed linear space $C$. For $a$ in $A$ we will write $a f$ for the image of $a$ under $f$. By $a b f$ we mean $(a b) f$. Suppose $\|a b f\| \leq$ $M\|a f\| \cdot\|b f\|$ for some real $M$, and all $a, b$ in $A$. Then we will say that $f$ is pseudo regular for $A$.

We study mainly the case when $C=A$ and $A$ is a commutative Banach algebra. We present some conditions which imply pseudo regularity, and some that prevent it. For example, if the non-zero elements of the spectrum of $f$ are bounded away from zero, then $f$ is pseudo regular. A result (5.3) in the other direction is that if $\sum_{-\infty}^{\infty}|t f(t)| d t<\infty$ for a pseudo regular element $f$ of $L^{1}(\mathbb{Z})$, then the spectrum is bounded away from 0 . Concerning the algebra $C^{1}[a, b]$, any $f$ which has no zero in common with its derivative is pseudo regular.

2. The relation to regularity. Behavior on extension. Let $A$ be a normed commutative algebra and let $f \in A$. One says that $f$ is subregular in $A$ if there is another commutative normed algebra $B$ which contains $A$ isomorphically, which has a unit 1, and in which the element corresponding to $f$ in $B$ has an inverse.

(2.1) Proposition. If $f$ is subregular in $A$, it is pseudo regular in $A$.

Proof. Let $f$ have the inverse $g$ in an algebra $B$ containing $A$. Let $a$ and $b$ belong to $A$. Then $\|a f b f g\|=\|a f b f g\| \leq\|a f\|\|b f\|\|g\|$, so $f$ is pseudo regular in $A$.

(2.2) Proposition. Pseudo regularity does not imply subregularity.

Here an example will suffice. Take $A$ to be the space $C(S, \mathbb{C})$ of continuous functions on some compact Hausdorff space $S$ with a non-trivial open-and-closed subset $E$. The characteristic function $e$ of $E$ satisfies $e^{2}=e$, so it cannot have an inverse in any $B$. Thus $e$ is not subregular. On the other hand, [A\&G, Theorem 3.3, or (3.22) below] shows that $e$ is pseudo regular. 
The next theorem is needed for our further examples, and comes close to giving the essence of pseudo regularity for algebras resembling function algebras. It is a restatement of [A\&G1, Th. 3.6].

(2.3) TheOREM [A\&G1, Th. 3.6]. Let $A$ be a function algebra. Let $f$ belong to $A$ and let $\Sigma$ be the set of values $f$ has on the Shilov boundary $\partial_{A}[\mathbf{G}, 10]$. If there is now a real $M$ such that $\left\|a^{2}\right\| \leq$ $M\|a f\|^{2}$ for all $a$ in $A$ then 0 is not a limit point of the rest of $\Sigma$. On the other hand, if 0 is not a limit point of the rest of $\Sigma$, then $f$ is pseudo regular.

(2.2) THEOREM. Let $f$ be an element of $A$, and let $A$ be a subalgebra of a normed algebra $B$. If $f$ is pseudo regular in $B$, it is pseudo regular in $A$; but not the other way around.

The first half is trivial, and for the second, an example will suffice. Let $A$ be the disc algebra $A(D)$ of those functions in $B=C(D, \mathbb{C})$ which are holomorphic in the interior of the disc $D$. Consider the complex variable $z$. It has an inverse (namely its complex conjugate) in the superalgebra $C\left(S^{1}, \mathbb{C}\right)$ of the disc algebra $A(D)$. By $(2.1)$, it is pseudo regular in $A$. But consider its spectrum as an element of $B=C(D, \mathbb{C})$. The Shilov boundary is the disc $D$, as is the set $\Sigma$, and 0 is a limit of the punctured disc. Hence, by (2.3), $f$ is not pseudo regular in $B$.

3. Strongly pseudo regular elements. An element $f$ of a normed algebra shall be called strongly pseudo regular if there exists a real number $M$ such that for every pair of elements $u, v$, of $A$, and every positive integer $n$, there hold the inequalities

$$
\left\|u v f^{n}\right\| \leq M^{n}\left\|u f^{n}\right\|\left\|v f^{n}\right\| .
$$

The reason for introducing this concept is not merely its connection with pseudo regularity, but also because it can be neatly characterized. ${ }^{1}$

(3.2) TheOREM. The statements (3.21), (3.22), (3.23) about an element of $f$ of a semisimple normed algebra $A$ with unit are equivalent. The statements (3.24) and (3.25) are equivalent to each other.

(3.21) $f$ is strongly pseudo regular,

\footnotetext{
${ }^{1}$ Professor Johnson has shown that strong pseudo regularity is not equivalent to pseudo regularity. See $[\mathbf{J}]$ or (3.5) below.

${ }^{2}[\mathrm{~L}, 62]$. We can do without the semi-simplicity by using the argument of Th. 3.4 below.
} 
(3.22) There is a superalgebra $B$ of $A$ which has an element $m$ such that $f=f^{2} m$;

(3.23) 0 is not a limit point of the rest of $\Sigma$.

(3.24) There is an element $m$ of $A$ such that $f=f^{2} m$,

(3.25) 0 is not a limit point of the rest of the spectrum of $f$.

Proof. Assume (3.21). Replace $u$ in (3.1) by $u^{n}$ and $v$ by $v^{n}$. Take the $n$th root of both sides, and using [G, 5.2], obtain $\left|u_{A} v_{A} f_{A}\right| \leq$ $M\left|u_{A} f_{A}\right|\left|v_{A} f_{A}\right|$. Here the heavy bars indicate the spectral norm and the suffix $A$ denotes the Gel'fand transform. This says that $F_{A}$ is pseudo regular as an element of the algebra of Gel'fand transforms. We refer to (2.3) and declare that (3.23) holds.

Next assume (3.23). Let $B$ be the algebra of all continuous complex valued functions on the Shilov boundary $S$. Define $m$ to be $1 / f$ where $f$ is not 0 , and 0 otherwise. Then $f^{2} m=f$, which shows (3.22); and (3.22) obviously implies (3.21).

Next, assume (3.25). Let $\sigma$ be the spectrum of $f$. Let $U_{1}$ be a neighborhood of the origin in the complex plane. Let $U_{2}$ be a neighborhood of $\sigma$ minus the origin. These $U_{i}$ can and shall be chosen to be disjoint, precisely because of (3.31). Let $\eta$ be a function which is 1 on the first set and 0 on the second. This function is holomorphic on a neighborhood of $\sigma$. We can use [G, 5.1 Theorem, 10] to obtain an element $e$ of $B$ which is 1 at the points of $S$ where $f$ is 0 , and 0 where $f$ is not 0 . Clearly $f e=0$. Moreover, the element $f+e$ is never 0 on the space of maximal ideals. So there is an $m$ in $A$ such that $m(f+e)=1$. Hence $f=m f^{2}$, which is (3.24).

A comparison of (3.2) and (2.3) shows that for an algebra $C(S, \mathbb{C})$, pseudo regularity implies strong pseudo regularity.

Another application, (3.3), of (3.2) shows the same for a convolution algebra. Let $G$ be a compact abelian group, and let $A$ be the algebra $L^{1}(G)$ of integrable ${ }^{3}$ complex valued functions on $G$, under convolution [L, 35D]. The space of maximal ideals is the character group $\Gamma$. Given an $f$ in $A$, it has a Gel'fand transform $f_{A}$ whose value at the point $m$ in $\Gamma$ is the Fourier coefficient [L, loc. cit.]

$$
f_{A}(m)=\int_{G} f(\theta) m(\theta) d \theta .
$$

\footnotetext{
${ }^{3}$ with respect to normalized Haar measure.
} 
(3.3) TheOREM. An $f$ in $L^{1}(G)$ for which

(3.31) only a finite number of Fourier coefficients are non-zero is strongly pseudo regular. Conversely, (3.31) holds if $f$ is pseudo regular.

Proof. Assume (3.31). Let $m$ belong to $\Gamma$. If the $m$ th Fourier coefficient of $f$ is $c_{m}$ and is not 0 , let the $m$ th Fourier coefficient of $g$ be the reciprocal of $c_{m}$. Otherwise let it be also zero. This clearly defines a linear combination $g$ of characters and thus an element of $L^{1}(G)$. It is easy to see that the Fourier transform of $f * f * g-f$ is 0 and hence that $f * f * g-f$ is 0 . By (3.22), $f$ is strongly pseudo regular.

Now suppose $f$ is pseudo regular. Then there is a real $M$ such that $\|u * u * f\| \leq M\|u * f\|^{2}$. Let $u$ be one of the characters $m[\mathbf{L}$, 38C]. Then $u * u=u$ and $\|u * f\|=\left|c_{m}\right|$. Call this positive number $C$. So $C(1-C M) \leq 0$. Thus if $C$ is not 0 then it is not less than $1 / M$, so of course there can be only finitely many $C$ not 0 , because Fourier transforms of $L^{1}$ functions vanish at infinity [L, 154-5].

We will go beyond (3.2) in two ways. In the first way, we consider algebras which are not semisimple. In the second, we enlarge $f$ to be a finite set of elements.

For the remainder of this section, let $A$ be a commutative Banach algebra with unit. For an element $a$ of $A$ there is the Gel'fand transform $a_{A}$, a function defined on the space of maximal ideals of $A$. It may happen that $a_{A}$ vanishes identically. Then $a$ is a radical element.

(3.4) Theorem. Let $A$ be as above and let $f$ be an element of $A$. Suppose that (as in (3.23))

0 is not a limit point of the spectrum $\sigma$ of $f$.

Then $f$ differs from a strongly pseudo regular element $g$ in $A$ by at most a radical element $r$.

Proof. Construct the open set $U_{i}$ and the function $\eta$ as above in the proof of (3.2).

(3.42) Let $\gamma$ be the function which is 0 on $U_{1}$ and $z$ on $U_{2}$. 
Then $\gamma+\eta$ is never 0 on the union $U$ of $U_{1}$ and $U_{2}$. Obviously there is a function $\mu$ holomorphic on $U$ such that $\mu(\gamma+\eta)=1$. Hence $\gamma \mu(\gamma+\eta)=\gamma$ and indeed

$$
\gamma \mu \gamma=\mu
$$

because $\gamma \eta=0$, as is easily verified.

We now apply the analytic-functional calculus as established in [A1, see 5.1, p. 427; G, ch. 3] The four functions $z, \gamma, \eta$, and $\mu$ give rise to four elements $f, g, e$, and $m$ of $A$ and they satisfy the relation $\mu g=g$ because the relation (3.43) is preserved under the functional calculus. Comparing this with (3.22), we see that $g$ is strongly pseudo regular.

We observe that $z-\gamma$ is 0 on the spectrum $\sigma$. Therefore $f_{A}-g_{A}$ is 0 on the space of maximal ideals whence $f-g$ is a radical element.

Thus (3.4) is established.

Professor B. E. Johnson has kindly communicated to me the next theorem, and its consequence (3.6). See [J].

(3.5) TheOREM ${ }^{4}$. Let $A$ be the Banach algebra $C^{1}[a, b]$. Let $f(t)=t$. Then $f$ is a pseudo regular element of $A$.

Proof. If 0 does not lie in $[a, b]$ then of course $f$ is pseudo regular, but in any case the following argument will work.

Let $J$ be the ideal of elements which vanish at 0 . Let $i$ belong to $J$. Define $q(i)$ as the function whose value is $i(t) / t$ for $t \neq 0$, and $i^{\prime}(0)$ otherwise. In this proof, let the supremum of the absolute value of any complex valued bounded function $h$ be denoted by $S(h)$. The norm $\|h\|$ of an element of $A$ is $S(h)+S\left(h^{\prime}\right)$.

By the theorem of the mean

$$
S(q(i)) \leq S\left(i^{\prime}\right) \leq\|i\| .
$$

If $j$ is another element of $J$ we have $S(q(i) j) \leq\|i\| \cdot\|j\|$.

We turn to $(q(i) j)^{\prime}$. Its value at $t \neq 0$ is $t q(i)^{\prime}(t)[j(t) / t]+$ $q(i)(t) j^{\prime}(t)$, and the obvious limit thereof for $t=0$. Thus $S\left((q(i) j)^{\prime}\right)$ $\leq S\left(t q(i)^{\prime}(t)\right) S[j(t) / t]+S(q(i)(t)) S\left(j^{\prime}(t)\right)$. Now $S[j(t) / t] \leq\|j\|$ by (3.51). So $S\left((q(i) j)^{\prime}\right) \leq S\left(t q(i)^{\prime}(t)\right)\|j\|+\|i\| \cdot\|j\|$. As to $t q(i)^{\prime}(t)$, it is $i^{\prime}(t)+i(q) / t$, so again by $(3.51), S\left(\operatorname{tq}(i)^{\prime}(t)\right) \leq S\left(i^{\prime}\right)+S\left(i^{\prime}\right)$ $\leq 2\|i\|$. Thus $S\left((q(i) j)^{\prime}\right) \leq 3\|i\| \cdot\|j\|$. Therefore

$$
\text { (3.52) } \quad\|q(i) j\| \leq 4\|i\| \cdot\|j\| \text {. }
$$

\footnotetext{
${ }^{4}$ generalized in (3.9) below.
} 
Take $i(t)$ to be $b(t) t$ where $b$ is any element of $A$. So $i=b f$. It is easy to verify that $q(i)=b$ itself, so $\|b j\| \leq 4\|b f\| \cdot\|j\|$. Now take $j=a f$, and obtain the assertion that $f$ is pseudo regular with $M \leq 4$.

(3.6) Corollary (B. E. Johnson). Pseudo regularity does not imply strong pseudo regularity.

Indeed, when 0 lies in the interval $[a, b]$, the $f$ above is not strongly pseudo regular by (3.23).

To this counterexample we may add another, namely (3.8) below. First another theorem.

(3.7) Theorem. Assume the hypotheses of (3.5), and take $[a, b]=$ $[0,1]$. Then $f^{2}$ is not pseudo regular.

Proof. We use the $S$-notation of (3.5), but we use the norm $N(h)=$ $|h(0)|+S\left(h^{\prime}\right)$ in $A$. This is equivalent to $\|\cdot\|$. We will study $f(t)=1-t$, rather than $t$. This helps in the notation. Assume $N\left(f^{2} g^{2}\right) \leq N\left(f^{2} g\right)^{2}$ with $g(t)=t^{n}$. We will estimate $N\left(f^{2} g\right)$, which is $S\left(\left(f^{2} g\right)^{\prime}\right)$. Now

$$
\left(f^{2} g\right)^{\prime}=\left[t^{n}-t^{n-1}\right][(n+2) t-n] .
$$

The extremal points of this expression are the two zeros of $(n+1)(n+2) t^{2}-2 n(n+1) t+(n-1) n$. We expand these roots in powers of $z=1 / n$. They are $t_{1,2}=1+\zeta z+\cdots$ where $\zeta=-2 \pm \sqrt{2}$. Inserting either of these into (3.71) gives an expression of the order of $z$, so

$$
N\left(f^{2} g\right) \text { is of the order of } z=1 / n \text {. }
$$

This implies that $N\left(f^{2} g^{2}\right)$ is of the order of $1 / 2 n$. So we get $K / n \leq$ $M(L / n)^{2}$ for all sufficiently large $n$. This forces $M$ to be infinite. In other words, $f^{2}$ couldn't have been pseudo regular.

(3.8) COROLlary. The product of pseudo regular elements need not be pseudo regular.

Corollary. Let $f$ belong to $A$ and suppose $f^{\prime}(x)$ is never 0 . Then $f$ is pseudo regular.

To prove this, just change the variable to $t=f(x)$, and use (3.5). 
So now we know that if either $f$ is never 0 in $C^{1}[a, b]$, or $f^{\prime}$ is never 0 , then $f$ is pseudo regular. In fact, we can generalize this and (3.5) in one theorem. ${ }^{5}$

(3.9) Theorem. Let $f$ belong to $C^{1}[a, b]$ and suppose $f$ and $f^{\prime}$ have no common zero. Then $f$ is pseudo regular.

Proof. We will adapt Johnson's line of reasoning as presented in (3.5). Let $J$ be the ideal elements of $C^{1}[a, b]$ which vanish on the set $Z=\left\{t_{1}, \ldots, t_{n}\right\}$ of zeros of $f$. For an element $i$ of $J$ we define $q(i)$ as $i(t) / f(t)$ or as $i^{\prime}(t) / f^{\prime}(t)$ according to whether $t$ is not, or is, a zero of $f$.

For each $k$ there is an open interval $V_{k}$ containing $t_{k}$ on which $f^{\prime}$ is bounded away from 0 . Let $V$ be the union of these $V_{k}$. Then $\left|f^{\prime}\right|>r$ in $V$ for some positive $r$. Moreover, $|f|>s$ for some positive $s$, outside of $V$. By multiplying $f$ by some constant, we can make sure that 1 will serve as $r$ and $s$.

Now suppose $t$ is outside of $V$. Then $|q(i)(t)| \leq|i(t)| /|f(t)| \leq$ $S(i)$.

Next suppose $t$ is in $V$. If $t$ is a zero of $f$ we have $|q(i)(t)|=$ $\left|i^{\prime}(t)\right| /\left|f^{\prime}(t)\right| \leq S\left(i^{\prime}\right)$. If $t$ is not a zero of $f$ then we can find a $z$ which is a zero and such that the interval $[z, t]$ lies in $V$, then

$$
q(i)(t)=\frac{i(t)-i(z)}{f(t)-f(z)}=\frac{i^{\prime}(v)}{f^{\prime}(v)}
$$

for some $v$ in $[z, t]$. Hence

$$
|q(i)(t)| \leq S\left(i^{\prime}\right) \text { for all } t \text { in } V .
$$

We can therefore assert that

$$
S(q(i)) \leq S(i)+S\left(i^{\prime}\right)=\|i\|, \quad \text { and } \quad S(q(i) j) \leq\|i\| \cdot\|j\|
$$

just as in (3.51). We now examine the $(q(i) j)^{\prime} . q(i) j$ is $i j / f$ off $Z$. Using Leibniz' rule yields $(q(i) j)^{\prime}=i^{\prime} q(j)+j^{\prime} q(i)-q(i) q(j) f^{\prime}$ on the (dense) complement of $Z$. Hence $S\left((q(i) j)^{\prime}\right) \leq S\left(i^{\prime}\right) S(q(j))+$ $S\left(j^{\prime}\right) S(q(i))-S(q(i)) S(q(j)) S\left(f^{\prime}\right) \leq\left(2+\left\|f^{\prime}\right\|\right)\|i\| \cdot\|j\|$, by a multiple use of (3.92). We must also consider the difference quotients where one or both points are on $Z$. The derivative there is easily found to be $i^{\prime} j^{\prime} / f^{\prime}$, since $j$ is 0 on $Z$. Hence

$$
\|q(i) j\|=S(q(i) j)+S\left((q(i) j)^{\prime}\right) \leq\left(3+\left\|f^{\prime}\right\|\right)\|i\| \cdot\|j\| .
$$

\footnotetext{
${ }^{5}$ Functions of several variables are discussed in $\S 7$ below.
} 
Now take $i=a f$ and $j=b f$, and conclude that $f$ is pseudo regular.

Statement (3.7) shows that when $f$ has a repeated zero, pseudo regularity may indeed fail.

\section{Pseudo regular systems.}

Definition. Let $F$ be a subset $\left\{f_{1}, \ldots, f_{N}\right\}$ of $A$. Let $A$ be a subalgebra of a second Banach algebra in which there exist elements $b_{1}, \ldots, b_{N}$ such that $f_{1} b_{1}+\cdots+f_{N} b_{N}=1$. Then $F$ is called $s u b$ regular.

Definition. Let $F$ be a subset $\left\{f_{1}, \ldots, f_{N}\right\}$ of $A$. For each $a$ in $A$ define $T_{F}(a)$ to be $\left\|f_{1} a\right\|+\cdots+\left\|f_{N} a\right\|$. Then $F$ is a pseudo regular system if there is a real constant $M$ such that for any $a, b$ in $A$ one has $T_{F}(a b) \leq M T_{F}(a) T_{F}(b)$.

Pseudo regular system is the same sort of generalization of regular system [A] as pseudo regular element is of regular element.

Just for the record, we state without proof the obvious analogue of (2.1).

(4.1) Proposition. If $F$ is subregular in $A$, it is pseudo regular in $A$.

More substantial is the analogue of (3.2).

(4.2) Theorem. Let $A$ be as above and let $F$ be a finite set $\left\{f_{1}\right.$, $\left.\ldots, f_{N}\right\}$ of elements of $A$. Suppose that

the origin $\mathbf{0}$ is not a limit point of the joint spectrum $\sigma$ of $F$.

Then $F$ differs from a pseudo regular system $G$ in $A$ by an additive $N$-tuple $\left(r_{1}, r_{2}, \ldots, r_{N}\right)$ where the $r_{i}$ are radical elements.

Proof. Find disjoint open sets $U_{1}$ and $U_{2}$ in complex $N$ space where $U_{1}$ contains the origin 0 and $U_{2}$ contains the rest of $\sigma$. Define a function $\eta$ to be 1 on $U_{1}$ and 0 on $U_{2}$. Using the analytic functional calculus gives us an idempotent $e$ such that $e_{A}$ is 1 when all the $f_{i A}$ are 0 , and 1 otherwise. Now $f_{i}=e f_{1}+(1-e) f_{i}$. Let $g_{i}=f_{i}(1-e)$. Then $f_{i}-g_{i}=e f_{i}$. Now $e_{A}$ is 0 when any of the $f_{i A}$ are not 0 and $f_{i A}$ is of course 0 when all the $f_{j A}$ are 0 . So $f_{i}-g_{i}$ is a radical element. 
I declare that the $N$ tuple $\left(e_{A}+\left(1-e_{A}\right) f_{1 A}, \ldots, e_{A}+\left(1-e_{A}\right) f_{N A}\right)$ have no common 0 . For when $e_{A}$ is 1 , then they are all 1 , and when $e_{A}$ is 0 they have the values of $\left(f_{1 A}, \ldots, f_{N A}\right)$, which are not all 0 when $e_{A}$ is 0 .

So the $g_{i}$ form a regular system, and there are elements $m_{i}$ such that $1=m_{1}\left(e+g_{1}\right)+\cdots+m_{N}\left(e+g_{N}\right)$. Fix a value of $j$ and obtain $g_{j}=\sum_{i} m_{i} g_{j} g_{i}$ because $g_{i} e=0$ for all $i$. Select any pair $a, b$ from $A$ and you have $g_{j} a b=\sum_{i} m_{i} g_{j} a g_{i} b$. Hence $\sum_{j} k\left\|g_{j} a b\right\| \leq$ $\sum_{i, j}\left\|g_{j} a\right\| \cdot\left\|g_{i} b\right\| M$, where $M$ is the greatest of the norms of the $m_{i}$. Thus the $g_{i}$ form a pseudo regular system.

5. Conditions preventing pseudo regularity. Let $A$ be a subalgebra of a function algebra $C(S, \mathbb{C})$ where $S$ is some compact Hausdorff space, and suppose that $A$ separates [G, pp. 15, 4] the points of $S$ and contains the unit.

REMARK. Let $f$ be an element of such an algebra $A$. Suppose $f$ is not pseudo regular in $A$. Then $f$ must vanish somewhere on the Shilov boundary $\partial_{A}$, or it is not pseudo regular.

(5.1) THEOREM. Let $f$ be a non-zero element of such an algebra, and suppose the Shilov boundary $\partial_{A}$ is connected. Then $f$ is pseudo regular if and only if it does not vanish on $\partial_{A}$.

For if $f$ does vanish on $\partial_{A}$ then the part of $\partial_{A}$ where $f$ is not 0 must be an open set $Z$. If $Z$ is empty then $f$ is 0 (and thus pseudo regular in a trivial way) or $\partial_{A}$ is not connected.

We now turn to a normed algebra with a norm other than the sup norm. Consider $A=L^{1}(\mathbb{Z})$ as an algebra under convolution

(5.2) Theorem. Let $f=\{c(n): n \in \mathbb{Z}\}$ belong to $L^{1}(\mathbb{Z})$ and suppose the Gel'fand transform series

$$
\sum_{n} c(n) e^{i n \theta}
$$

never vanishes on the unit circle. Then $f$ is pseudo regular.

We make this well-known statement only to draw attention to the converse. If (5.21) is sometimes 0 , must it be not pseudo regular? We can almost prove it. ${ }^{6}$ Let $\|\cdot\|$ denote the usual norm in $A=L^{1}(\mathbb{Z})$. The operations are linear combination and convolution. We write simply $f g$ for the convolution of $f$ and $g$. 
(5.3) Theorem [Compare A\&G2, 3.1]. Let $f \in L^{1}(\mathbb{Z})$ and suppose

$$
\sum_{-\infty}^{\infty}|t f(t)| d t<\infty,
$$

(5.32) $\left\|u^{2} f\right\| \leq M\|u f\|^{2}$ for some real $M$ and at least for all $u$ of finite support for which $u(t)=0$ when $t<0$.

Then $f$ has an inverse in $L^{1}(\mathbb{Z})$ or $f=0$.

We insert two lemmas. We omit the proof of the first.

(5.4) Lemma. Let $s(p)=\sum_{-\infty}^{p} f(t) d t$. Let $N$ be a positive integer. Define $u$ by setting $u(t)$ be 1 when $t$ lies in the interval $[0, N]$, and 0 otherwise. Then

$$
u f(t)=s(t)-s(t-N-1) .
$$

(5.5) Lemma. Let $J=\sum_{-\infty}^{0}|t f(t)| d t$. Let

$$
D=f^{\wedge}(0)=\sum_{-\infty}^{\infty} f(t) d t
$$

where $f^{\wedge}$ is the Fourier transform of $f$. Let $K=\sum_{0}^{\infty}|t f(t)| d t$. Then

$$
\sum_{-\infty}^{0}|s(t)| d t \leq J
$$

and

$$
\sum_{0}^{\infty}|s(t)-D| d t \leq K .
$$

These are readily obtained by reversing the order of summation. To derive (5.52) one starts by observing that

$$
s(p)+\sum_{p+1}^{\infty} f(t)=D .
$$

Define $T$ by

$$
T=\sum_{-\infty}^{\infty}|t f(t)|
$$

Then $J+K=T$.

(5.55) Define $h(t)$ to be 0 for $t<0$ and 1 for all other values. 
Proposition. According to (5.41), $u f=s-s^{N-1}$ where $s^{N+1}$ is $s$ shifted $N+1$ units to the right. As to its norm,

$$
\|u f\| \leq(N+1)|D|+2 T .
$$

Proof. $s-s^{N+1}=s-D h+D h-D h^{N+1}+D h^{N+1}-s^{N+1}$. Therefore $\left\|s-s^{N+1}\right\| \leq\|s-D h\|+\left\|D h-D h^{N+1}\right\|+\left\|D h^{N+1}-s^{N+1}\right\|$.

Now $\|s-D h\|=\left\|D h^{N+1}-s^{N+1}\right\|$ which is not greater than $J+K$ by (5.51) and (5.52). The term $\left\|D h-D h^{N+1}\right\|=(N+1)|D|$. From this, (5.6) follows.

We resume the proof of (5.3) by deducing from this that the right side of the inequality in (5.32) is $M[(N+1)|D|+2 T]^{2}$, and turn to the left side.

A real number $\alpha$ represents a point of the space of maximal ideals of $A$. The value of the Gel'fand transform of $f$ there is $f^{\wedge}(\alpha)$. This has to be numerically at most equal to the norm of $u^{2} f$, and hence, assuming (5.32),

$$
\left|u^{\wedge}(\alpha)^{2}\right|\left|f^{\wedge}(\alpha)\right| \leq M[(N+1)|D|+2 T]^{2} .
$$

Let us evaluate this for $\alpha=0$, noting $u^{\wedge}(0)=N+1$. So $(N+1)^{2}|D| \leq$ $M[(N+1)|D|+2 T]^{2}$. Since $N$ is arbitrary, we have $|D| \leq M|D|^{2}$. Thus either $f^{\wedge}(0)=0$, or $1 / M \leq\left|f^{\wedge}(0)\right|$.

The property of pseudo regularity has the invariance property that for each character $\alpha, e^{-i \alpha t} f(t)$ is pseudo regular if $f$ is. Thus we either have

$$
f^{\wedge}(\alpha)=0,
$$

or

$$
1 / M \leq\left|f^{\wedge}(\alpha)\right| .
$$

If (5.7) holds for some $\alpha$, it must hold for all, because $f^{\wedge}$ is continuous, and $f$ must be 0 . If (5.8) holds then $f^{\wedge}$ does not vanish anywhere on the space of maximal ideals, and hence $f$ has an inverse.

It almost goes without saying that the converse is true, too.

6. The situation based on the action of $L^{1}(\mathbb{Z})$ on $L^{2}(\mathbb{Z})$. A $u \in$ $L^{1}(\mathbb{Z})$ works on an element $f$ in $L^{2}(\mathbb{Z})$ by sending it into $u * f$ in $L^{2}(\mathbb{Z})$. So we are defining $u f$ as $u * f$ in this situation. Then $f$ is pseudo regular if there is a real $M$ such that $\|(u * v) * f\| \leq$ $M\|u * f\|\|v * f\|$ where the norm is that of $L^{2}(\mathbb{Z})$. 
(6.1) TheOREM. Let $f \in L^{1}(\mathbb{Z})$ and suppose

$$
\sum_{-\infty}^{\infty}|t f(t)| d t<\infty
$$

(6.12) $\left\|u^{2} f\right\| \leq M\|u f\|^{2}$ for some real $M$ and at least for all those $u$ in $L^{2}(\mathbb{Z})$ for which $u(t)=0$ when $t<0$.

Then $f=0$.

Proof. The norm $\|\cdot\|$ shall now refer to $L^{2}(\mathbb{Z})$. Define $D, h, u$ and $s$ as in (5.3)-(5.55). We want an upper estimate for $\left\|s-s^{N+1}\right\|$. $\left\|s-s^{N+1}\right\| \leq\|s-D h\|+\left\|D h-D h^{N+1}\right\|+\left\|D h^{N+1}-s^{N+1}\right\|$. Now $\left\|D h^{N+1}-s^{N+1}\right\|=\|s-D h\|$, and our next step is to show that

$$
\|s-D h\| \quad \text { is finite. }
$$

$$
\begin{aligned}
\|s-D h\|^{2} & =\sum_{p=-\infty}^{-1}|s(p)|^{2}+\sum_{p=0}^{\infty}|s(p)-D|^{2} \\
& =\sum_{p=-\infty}^{-1}\left|\sum_{t=-\infty}^{p} f(t)\right|^{2}+\sum_{p=0}^{\infty}\left|\sum_{t=p+1}^{\infty} f(t)\right|^{2} \\
& \leq \sum_{p=-\infty}^{-1}\left|\sum_{t=-\infty}^{p} f_{t}\right|^{2}+\sum_{p=0}^{\infty}\left|\sum_{t=p+1}^{\infty} f_{t}\right|^{2}
\end{aligned}
$$

where $f_{t}$ is an abbreviation for $|f(t)|$. Let these two terms be called $S_{1}$ and $S_{2}$ respectively, for a moment. Take $S_{1}$ and let the index $p$ be called $-q$. Then

$$
S_{1}=\sum_{q=1}^{\infty}\left|\sum_{t=q}^{\infty} f_{-t}\right|^{2}
$$

which we will call $T^{-}$. Concerning $S_{2}$ we can surely say $S_{2} \leq$ $\sum_{q=1}^{\infty}\left|\sum_{t=q}^{\infty} f_{t}\right|^{2}$ which sum we shall call $T^{+}$. We may rewrite $T^{+}$as $\sum_{q=1}^{\infty} \sum_{t=q}^{\infty} \sum_{u=q}^{\infty} f_{t} f_{u}$. This is twice the sum over all lattice points for which $u \geq t \geq q \geq 0$. Thus

$$
T^{+}=2 \sum_{u \geq t \geq 0} \sum_{q=0}^{t} f_{t} f_{u} \sum_{u \geq t \geq 0}(t+1) f_{t} f_{u} \leq 2(T+U) U
$$


where $T$ is given in (5.54), and $U$ is the $L^{1}$ norm of $f$. The reason for the $\leq$ is that in the summing for $T^{+}$only the values of $f_{t}$ with nonnegative suffix are used, whereas in $U$ all suffixes are involved.

It is easy to see that $T^{-}$also is not greater than $2(T+U) U$, and consequently (6.13) is true.

Concerning $\left\|D h-D h^{N+1}\right\|$ it is easy to see that it is equal to $|D| \sqrt{N+1}$. This gives the dominant term on the right side of the inequality $\left\|u^{2} f\right\| \leq M\|u f\|^{2}$ in (6.12). Squaring both sides it implies $\left\|u^{2} f\right\| \leq(b+|D| \sqrt{N+1})^{2}$. By Parseval,

$$
\left\|u^{2} f\right\|^{2}=(1 / 2 \pi) \int_{-\pi}^{\pi}\left|u^{\wedge}\right|^{4}\left|f^{\wedge}\right|^{2} d \theta
$$

It is not hard to compute that $(1 / 2 \pi) \int_{-\pi}^{\pi}\left|u^{\wedge}\right|^{4} d \theta$ is a polynomial $N_{3}$ of degree 3 in $N$, and that $\left|u^{\wedge}\right|^{4} / N_{3}$ satisfy the conditions (i), (ii), (iii) of $[\mathrm{Z}, 3.201]$ associated with the concept of an approximate identity. Therefore $(1 / 2 \pi) \int_{-\pi}^{\pi} p\left(1 / N_{3}\right)\left|u^{\wedge}\right|^{4}\left|f^{\wedge}\right|^{2} d \theta$ tends to $\left|f^{\wedge}(0)\right|^{2}$ as $N$ goes to $\infty$. But $(b+|D| \sqrt{N+1})^{2} / N_{3}$ tends to 0 , so $f^{\wedge}(0)=0$.

Appealing again to the invariance which led to (5.7), we conclude that $f=0$.

One might wonder what about (5.8). Apparently regular elements are not pseudo regular in this situation. If the identity element of $L^{1}(\mathbb{Z})$ were pseudo regular, then $L^{2}(\mathbb{Z})$ would be a Banach algebra.

7. $C^{1}$ algebras of functions of several variables. The general idea is that if the differential $d f$ is not 0 anywhere on the set $Z$ of zeros of $f$, then $f$ should be pseudo regular. In order to prove any theorems, we have to augment this hypothesis with some technical details which cannot be overlooked. We will assume that $M$ is a Riemannian manifold, or a closed interval in some $\mathbb{R}^{m}$, and consider the algebra of $C^{\prime}$ functions on $M$. For any numerical valued function $f$ on $M$ we define $S(f)$ to be the sup of the values $|f(t)|$ for $t$ in $M$. If $f$ is $C^{1}$, we denote by $f^{\prime}$ the gradient of $f$. Let $\left|f^{\prime}\right|$ be the length of $f^{\prime}$ and abbreviate $S\left(\left|f^{\prime}\right|\right)$ by $S\left(f^{\prime}\right)$. We consider the algebra of those $C^{1}$ functions $f$ for which $\|f\|=S(f)+S\left(f^{\prime}\right)$ is finite.

We will now define an $f^{\prime}, u$ curve in $M$, where $u>0$. It is a $C^{1}$ curve with tangents $T$ such that $T \cdot f^{\prime}>u|T| \cdot\left|f^{\prime}\right|$.

Let $V^{r}$ be the set of points where $|f|<r$.

We now enumerate the precise conditions imposed on $f$.

(7.1) There are $r, s>0$ such that $\left|f^{\prime}\right|>s$ on $V^{r}$. 
(7.2) There is a $u>0$ such that given any point $t$ of $V^{r}$, there is an $f^{\prime}, u$ curve lying in $V^{r}$ and connecting $t$ to $Z .^{7}$

Let $f$ satisfy these. It is enough to treat the case $r=s=1$. We will abbreviate $V^{1}$ to $V$. We will follow the line of reasoning of (3.9). Let $J$ be the ideal of elements of $C^{1}[M]$ which vanish on the set $Z$ of zeros of $F$. Let $i$ belong to $J$. We define $q(i)$ as

$$
i(t) / f(t)
$$

or as

$$
i^{\prime}(t) \cdot f^{\prime}(t) / f^{\prime}(t) \cdot f^{\prime}(t)
$$

according to whether $t$ is not, or is, a zero of $f$. (7.31) obviously defines a function continuous on $Z$. It is not hard to show that (7.3) approaches (7.31) as $t$ approaches a point of $Z$. So $q$ is continuous on $M$.

Now suppose $t$ is outside of $V$. Then $|q(i)(t)| \leq|i(t)| /|f(t)| \leq$ $S(i)$. If $t$ is in $Z$, we can see from (7.31) that $|q(i)(t)| \leq\left|i^{\prime}(t)\right| \leq$ $S\left(i^{\prime}\right) \leq\|i\|$ for such $t$.

Now suppose $t$ is in $V$. Then we can find an $f^{\prime}, u$ curve $c$ leading from $z$ in $Z$ to $t, c$ lying in $V$. Now $i(t)=\int i^{\prime} \cdot T d s$, where the integral is over $c, T$ is the unit tangent to $c$, and $s$ is the arc length. By the theorem of the mean, $i(t)=i^{\prime} \cdot T s$, where now $i^{\prime} \cdot T$ is evaluated somewhere along $c$, so inside $V$. The same thing holds for $f$. Now $\left|i^{\prime} \cdot T\right| \leq S\left(i^{\prime}\right) \leq\|i\|$, and $u S\left(f^{\prime}\right)|\leq| f^{\prime} \cdot T \mid$, so

$$
|q(i)(t)| \leq\|i\| \text {. }
$$

We can therefore assert that

$$
S(q(i)) \leq S(i)+S\left(i^{\prime}\right)=\|i\|, \quad \text { and } \quad S(q(i) j) \leq\|i\| \cdot\|j\| .
$$

We now examine the $(q(i) j)^{\prime} . q(i) j$ is $i j / f$ off $Z$. Using Leibniz' rule yields $(q(i) j)^{\prime}=i^{\prime} q(j)+j^{\prime} q(i)-q(i) q(j) f^{\prime}$ on the (dense) complement of $Z$. Hence $S\left((q(i) j)^{\prime}\right) \leq S\left(i^{\prime}\right)(q(j))+S\left(j^{\prime}\right) S(q(i))-$ $S(q(i)) S(q(j)) S\left(f^{\prime}\right) \leq\left(2+\left\|f^{\prime}\right\|\right)\|i\| \cdot\|j\|$, by a multiple use of (7.34). We must also consider the difference quotients where one or both points are on $Z$, on which $j$ is 0 . The derivative there is

\footnotetext{
${ }^{7}$ Let $M$ be the closed first quadrant in $\mathbb{R}^{2}$ and let $f=x^{2}-y$. Then for each $u,(7.2)$ does not hold for $t=(0, v)$ when $v$ is sufficiently small. It fails because $Z$ is tangent to the boundary of $M$.
} 
$\left(i^{\prime} \cdot f^{\prime} / f^{\prime} \cdot f^{\prime}\right) j^{\prime}$. Hence

(7.35) $\quad\|q(i) j\|=S(q(i) j)+S\left((q(i) j)^{\prime}\right) \leq\left(3+\left\|f^{\prime}\right\|\right)\|i\| \cdot\|j\|$.

Now take $i=a f$ and $j=b f$, and observe that $f$ is pseudo regular.

\section{REFERENCES}

[A\& G] R. F. Arens and M. Goldberg, Multiplicativity factors for seminorms, J. Math. Anal. Appl., 146 (1990), 469-481.

[A\& G1] _ A class of seminorms on function algebras, to appear in the same J. Math. Anal. Appl.

[A\&G2] $\quad M$ factors for $L^{1}(\mathbb{Z})$, in preparation.

[A\& K] R. F. Arens and I. Kaplansky, Topological representation of algebras, Trans. Amer. Math. Soc., 63 (1948), 457-481.

[A] R. F. Arens, Dense inverse limit rings, Michigan Math. J., 5 (1958), 169-182.

[A1] - The analytic-functional calculus in commutative topological algebras, Pacific J. Math., 11 (1961), 405-429.

[G] T. W. Gamelin, Uniform Algebras, Prentice-Hall, Inc., 1969.

[J] B. E. Johnson, Pseudo regular elements and the auxiliary multiplication they induce, to appear Pacific J. Math.

[L] Lynn H. Loomis, An Introduction to Abstract Harmonic Analysis, Van Nostrand, Princeton, 1963.

[Z] Antoni Zygmund, Trigonometrical Series, 1935.

Received March 4, 1991 and in revised form May 15, 1991.

UNIVERSITY OF CALIFORNIA

Los ANGeles, CA 90024-1555 



\section{PACIFIC JOURNAL OF MATHEMATICS EDITORS}

V. S. VARAdARAJAN

(Managing Editor)

University of California

Los Angeles, CA 90024-1555

Herbert Clemens

University of Utah

Salt Lake City, UT 84112

F. Michael CHRIST

University of California

Los Angeles, CA 90024-1555

ThOMAs ENRIGHT

University of California, San Diego

La Jolla, CA 92093
Nicholas ERCOLANI

University of Arizona

Tucson, AZ 85721

R. FINN

Stanford University

Stanford, CA 94305

VAUGHAN F. R. JoNES

University of California

Berkeley, CA 94720

SteVen KercKhofF

Stanford University

Stanford, CA 94305
C. C. MOORE

University of California

Berkeley, CA 94720

MARTIN SCHARLEMANN

University of California

Santa Barbara, CA 93106

HAROLD STARK

University of California, San Diego

La Jolla, CA 92093

\section{ASSOCIATE EDITORS}
R. Arens
E. F. BeCKenBACH
B. H. NeumanN
F. WolF
(1904-1989)
K. YoshidA (1906-1982)

TIONS

UNIVERSITY OF ARIZONA

UNIVERSITY OF BRITISH COLUMBIA

UNIVERSITY OF OREGON

UNIVERSITY OF SOUTHERN CALIFORNIA

CALIFORNIA INSTITUTE OF TECHNOLOGY

UNIVERSITY OF CALIFORNIA

MONTANA STATE UNIVERSITY

STANFORD UNIVERSITY

UNIVERSITY OF HAWAII

UNIVERSITY OF NEVADA, RENO

UNIVERSITY OF TOKYO

NEW MEXICO STATE UNIVERSITY

UNIVERSITY OF UTAH

OREGON STATE UNIVERSITY

WASHINGTON STATE UNIVERSITY

UNIVERSITY OF WASHINGTON 


\section{Pacific Journal of Mathematics}

Vol. 154, No. $1 \quad$ May, 1992

Richard Arens, Pseudo regular elements in a normed ring $\ldots \ldots \ldots \ldots \ldots 1$

Joan Birman and William W. Menasco, Studying links via closed braids.

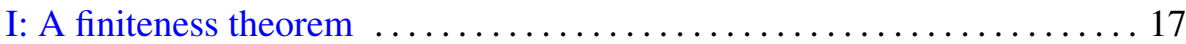

Etsurō Date, Michio Jimbo, Kei Miki and Tetsuji Miwa, Braid group

representations arising from the generalized chiral Potts models ....... 37

Toshihiro Hamachi, A measure theoretical proof of the Connes-Woods

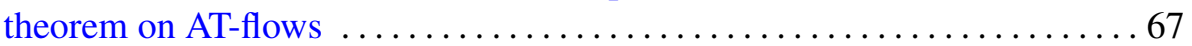

Allen E. Hatcher and Ulrich Oertel, Affine lamination spaces for surfaces ....................................... 87

David Joyner, Simple local trace formulas for unramified $p$-adic groups $\ldots .103$

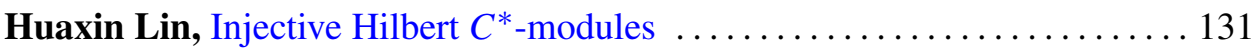

John Marafino, The boundary of a simply connected domain at an inner

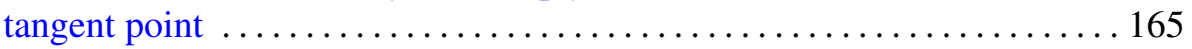

Gonzalo Riera and Rubi Rodriguez, The period matrix of Bring's curve . . 179 